\title{
Prognostic and Therapeutic Significance of Carbohydrate Antigen 19-9 as Tumor Marker in Patients with Pancreatic Cancer
}

\author{
Stefan Boeck ${ }^{\mathrm{a}}$ Petra Stieber $^{\mathrm{b}}$ Stefan Holdenrieder ${ }^{\mathrm{b}}$ Ralf Wilkowski $^{\mathrm{c}}$ \\ Volker Heinemann ${ }^{a}$

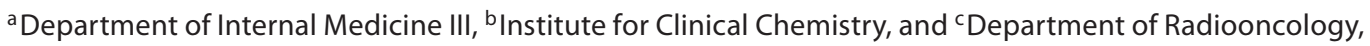 \\ Klinikum Grosshadern, Ludwig Maximilians University of Munich, Munich, Germany
}

\section{Key Words}

Pancreatic cancer - Tumor marker • Carbohydrate

antigen 19-9

\begin{abstract}
In pancreatic cancer (PC) accurate determination of treatment response by imaging often remains difficult. Various efforts have been undertaken to investigate new factors which may serve as more appropriate surrogate parameters of treatment efficacy. This review focuses on the role of carbohydrate antigen 19-9 (CA 19-9) as a prognostic tumor marker in PC and summarizes its contribution to monitoring treatment efficacy. We undertook a Medline/PubMed literature search to identify relevant trials that had analyzed the prognostic impact of CA 19-9 in patients treated with surgery, chemoradiotherapy and chemotherapy for PC. Additionally, relevant abstract publications from scientific meetings were included. In advanced PC, pretreatment CA 19-9 levels have a prognostic impact regarding overall survival. Also a CA 19-9 decline under chemotherapy can provide prognostic information for median survival. A $20 \%$ reduction of CA 19-9 baseline levels within the first 8 weeks of chemotherapy appears to be sufficient to define a prognostic relevant subgroup of patients ('CA 19-9 responder'). It still
\end{abstract}

remains to be defined whether the CA 19-9 response is a more reliable method for evaluating treatment efficacy compared to conventional imaging.

Copyright $\odot 2006$ S. Karger AG, Basel

\section{Introduction}

Pancreatic cancer (PC) is a disease still characterized by its unfavorable outcome. The overall median survival is 3-5 months with a 1-year survival rate of less than $10 \%$ [1]. More than $80 \%$ of patients are initially diagnosed in an advanced stage of disease, where the potential curative resection is no longer possible. Therefore, in most of the patients the first treatment goal is control of disease-related symptoms and prolongation of survival. Known prognostic factors for survival are the stage of disease, degree of tumor cell differentiation, weight loss, performance status and primary tumor site $[2,3]$.

The diagnosis of PC is usually established based on imaging techniques like ultrasound, computerized tomography (CT) or magnetic resonance imaging and a subsequent histological confirmation. A known phenomenon that complicates tumor imaging (especially in CT scan) is the desmoplastic stroma reaction induced by

\section{KARGER}

Fax +4161306 1234 E-Mail karger@karger.ch www.karger.com
(C) 2006 S. Karger AG, Basel

$0030-2414 / 06 / 0704-0255 \$ 23.50 / 0$

Accessible online at:

www.karger.com/ocl
Dr. Stefan Boeck, Department of Internal Medicine III

Klinikum Grosshadern, Ludwig Maximilians University of Munich

Marchioninistrasse 15, DE-81377 Munich (Germany)

Tel. +49897095 2208, Fax +49897095 5256

E-Mail stefan.boeck@med.uni-muenchen.de 
Table 1. Prognostic value of pretreatment CA 19-9

\begin{tabular}{|c|c|c|c|}
\hline Authors & $\mathrm{n}$ & $\begin{array}{l}\text { CA } 19-9 \text { cutoff } \\
\text { level, } \mathrm{U} / \mathrm{ml}\end{array}$ & Median survival, months \\
\hline Lundin et al. [16], 1994 & 160 & $370^{\mathrm{a}}$ & 9.5 vs. $4.4(\mathrm{p}<0.01)$ \\
\hline Sperti et al. [17], 1993 & 30 & $200^{\mathrm{a}}$ & 22.0 vs. $8.0(\mathrm{p}<0.001)$ \\
\hline Katz et al. [28], 1998 & 104 & $680^{\mathrm{b}}$ & 20.0 vs. $8.0(p=0.0003)$ \\
\hline Ikeda et al. [31], 2001 & 55 & $1,000^{\mathrm{b}}$ & 10.3 vs. $7.2(p=0.04)$ \\
\hline Micke et al. [32], 2003 & 95 & $420^{\mathrm{b}}$ & 12.3 vs. $7.1(p=0.0056)$ \\
\hline Saad et al. [47], 2002 & 28 & $1,212^{\mathrm{c}}$ & 14.9 vs. $7.4(p=0.0013)$ \\
\hline Maisey et al. [52], 2005 & 154 & $958^{\mathrm{c}}$ & 11.2 vs. $5.5(p=0.0004)$ \\
\hline
\end{tabular}

the tumor itself in surrounding soft tissue [4]. Often, this desmoplastic reaction makes it difficult to differentiate normal pancreas, local inflammation and fibrosis from malignant tissue and therefore imaging may sometimes be inaccurate in the assessment of treatment response. Thus, other clinical endpoints and surrogate markers of treatment efficacy like the term 'clinical benefit response' were created (defined as a composite endpoint consisting of pain, analgesic consumption, performance status and weight [5]), which may be more appropriate than assessment of tumor diameters. Furthermore, there have also been studies that try to establish a definition of treatment response according to changes in the levels of serum tumor markers like carbohydrate antigen 19-9 (CA 19-9).

CA 19-9 is a tumor-associated antigen (first described by Koprowski et al. $[6,7]$ ) defined by a monoclonal antibody (1116 NS 19-9) which was produced by a hybridoma prepared from mouse spleen, immunized with a human colorectal carcinoma cell line. CA 19-9 is the sialylated Lewis (Le) $)^{\mathrm{a}}$ blood group antigen and individuals with an $\mathrm{Le}^{\mathrm{a}-\mathrm{b}-}$ phenotype (lacking the Lewis antigen glycosyltranferase) are unable to synthesize CA 19-9 [8]. The diagnostic value in patients with suspected PC was evaluated in several studies, showing a sensitivity and specificity of the CA $19-9$ assay of about 70 and $80 \%$, respectively [9-12]. In addition, serial changes in CA 19-9 levels can also provide useful diagnostic information in patients with PC [13]. Several reports have shown a significant correlation of CA 19-9 serum levels with different stages of disease and resectability of the tumor [11, $14,15]$. In about $60-80 \%$ of patients with locally advanced or metastatic disease elevated CA 19-9 levels are found.

Nevertheless, a definite role of CA 199 as a surrogate marker for treatment efficacy in the clinical course of pa- tients with PC has not yet been defined. Especially the interpretation of serial tumor marker kinetics during therapy is uncertain and needs further validation. The focus of this review article is based on the prognostic value of serum CA 19-9 levels, with special regard to the impact of CA 19-9 changes during therapy on treatment outcome.

\section{Prognostic Value of CA 19-9 in Patients Undergoing Surgery for PC}

\section{Importance of Preoperative CA 19-9}

The association between serum tumor marker levels and outcome was first established in patients undergoing surgery for pancreatic adenocarcinoma. A significant difference in survival was shown between patients with preoperative CA 19-9 levels higher or lower than $370 \mathrm{U} /$ $\mathrm{ml}$ (4.4 vs. 9.5 months, $\mathrm{p}<0.01$ ), if the stage of the disease was not taken into account. A statistical difference in median survival was found only in patients with stage II and III disease and a CA 19-9 cutoff level of $370 \mathrm{U} / \mathrm{ml}$ [16]. Also, with a preoperative CA 19-9 cutoff level of $200 \mathrm{U} /$ $\mathrm{ml}$, significant differences in median survival between the two groups were demonstrated (22 vs. 8 months, [17]) (table 1).

\section{Importance of Postoperative CA 19-9}

Similar to these results, postoperative CA 19-9 levels appear to have an influence on survival in patients with PC. In the long-term follow-up of 75 patients after curative resection for pancreatic ductal adenocarcinoma, high postoperative CA 19-9 levels were - beside adjuvant treatment, $\mathrm{T}$ sage and nodal status - a significant prognostic 
Table 2. Impact of CA19-9 response to CRT on survival

\begin{tabular}{|c|c|c|c|c|}
\hline \multirow[t]{2}{*}{ Authors } & \multirow[t]{2}{*}{$\mathrm{n}$} & \multirow{2}{*}{$\begin{array}{l}\text { Definition of response } \\
\text { (CA 19-9 reduction), \% }\end{array}$} & \multicolumn{2}{|c|}{ Median overall survival, months } \\
\hline & & & responder & nonresponder \\
\hline Katz et al. [28], 1998 & 104 & $>75^{\mathrm{a}}$ & 23.0 & $8.0(\mathrm{p}=0.003)$ \\
\hline Okusaka et al. [29], 1998 & 34 & $>50^{\mathrm{b}}$ & 10.6 & 4.1 (p: n.a.) \\
\hline Micke et al. [32], 2003 & 59 & decreasing $\mathrm{b}, \mathrm{c}$ & 13.9 & $6.0(p=0.0002)$ \\
\hline \multicolumn{5}{|c|}{$\begin{array}{l}\text { n.a. = Not available. } \\
\text { a Patients undergoing neoadjuvant, adjuvant and palliative CRT. } \\
\text { b Patients receiving CRT for locally advanced PC. } \\
\text { c Patients with decreasing CA 19-9 levels during treatment. }\end{array}$} \\
\hline
\end{tabular}

factor for overall survival in univariate analysis [18]. Patients whose elevated postresection CA 19-9 values normalized $(<37 \mathrm{U} / \mathrm{ml})$ after surgical resection not only had a longer median overall survival but were also characterized by a prolonged disease-free survival [19]. The observation that normalizing CA 19-9 levels (usually defined as $<37 \mathrm{U} / \mathrm{ml}$ ) after surgery are a significant prognostic factor for overall survival was confirmed by other groups as well $[15,17,20,21]$. The decline of elevated CA 19-9 levels back to a normal range after surgery may also be stage dependent: one study showed that in patients with stage I, II and III of disease, CA 19-9 values normalized only in 29,13 and $10 \%$ of patients, respectively [20].

\section{Neoadjuvant Chemoradiotherapy}

In the neoadjuvant treatment setting for potentially resectable PC, a significant correlation between disease control and CA 19-9 decrease after 5-fluorouracil (5$\mathrm{FU}$ )-based chemoradiotherapy (CRT) was found by Willet et al. [22]. In their study, only $21 \%$ of patients with declining CA 19-9 levels after irradiation demonstrated metastases or local tumor progression whereas $90 \%$ of patients with increasing CA 19-9 values had disease progression. Pretreatment serum CA 19-9 levels before neoadjuvant CRT may, furthermore, play a role in predicting resectability of the tumor and survival as indicated by a retrospective analysis in 86 patients [23].

\section{Prognostic Impact of CA 19-9 Kinetics}

Another possible option for studying the prognostic value of CA 19-9 is the analysis of changes in its time course (e.g. doubling time) during the clinical course of patients with PC. A significant correlation between CA 19-9 doubling time and median survival was found in patients with inoperable PC and in patients with pallia- tive operation, whereas in patients with recurrence no correlation was noted [24]. Already Tian et al. [21] have reported a significant linear correlation $(\mathrm{r}=0.5)$ between survival time and doubling time of serum CA 19-9 levels in 15 patients undergoing surgery, indicating that CA 19-9 kinetics may be an additional useful tool for obtaining prognostic information in the clinical course of a patient with PC.

\section{CA 19-9 and CRT}

Radiation therapy and specifically combined CRT are effective treatment options in the multidisciplinary treatment of PC. Besides neoadjuvant treatment approaches, particularly adjuvant CRT after surgery and primary CRT for locally advanced disease were studied in several clinical trials [25-27]. In a retrospective analysis of a heterogeneous collective of 104 patients (undergoing neoadjuvant, adjuvant and palliative CRT), CA 19-9 levels at the time of diagnosis were found to be a significant prognostic indicator on univariate and on multivariate analysis. Patients with pretreatment values greater than the median of $680 \mathrm{U} / \mathrm{ml}$ had a survival of only 8 months compared to patients with levels below $680 \mathrm{U} / \mathrm{ml}$, having a median survival of 20 months (table 1). Also the posttreatment nadir of CA 19-9 showed a significant association with median survival. Katz et al. [28] additionally demonstrated that patients with a decrease of CA 19-9 levels $>75 \%$ (in response to treatment) had a statistically significant longer survival rate compared to patients with a CA 19-9 decline of $<75 \%$ (table 2 ).

\section{CA 19-9 and CRT for Locally Advanced Disease}

In patients with locally advanced and unresectable PC, a biochemical treatment response monitored by se- 
rum CA 19-9 levels seems to have a prognostic impact on survival. Okusaka et al. [29] defined a CA 19-9 responder under radiotherapy for unresectable disease as a patient whose serum CA 19-9 level was reduced by more than $50 \%$ of the pretreatment baseline level after treatment. Out of 34 patients in their analysis $21 \%$ were classified as CA 19-9 responder. These responders had a longer median survival after radiation therapy than nonresponders (table 2). There was also a relationship between objective response and tumor marker response in this study. In the group of CA 19-9 responders, none of the 6 patients had progressive disease during the observation period, and all 5 patients with tumor progression were classified as CA 19-9 nonresponders. Similar to this observation, Wilkowski et al. [30] defined a (biochemical) partial response as a decline of $>50 \%$ in CA $19-9$ serum levels in 171 patients under CRT for locally advanced disease. In this trial, the biochemical response status had no impact on overall survival and also the pretreatment CA 19-9 values did not predict response or progression-free survival. Only patients without elevated CA 19-9 serum levels had a significantly longer progression-free and overall survival.

Regarding the significance of baseline CA 19-9 levels in patients receiving CRT, Ikeda et al. [31] identified the pretreatment CA 19-9 value to be a significant prognostic factor in univariate (see table 1 ) as well as in multivariate (HR 2.17, $\mathrm{p}=0.02$ ) analysis of 55 patients with locally advanced PC undergoing treatment with 5-FU- or cisplatin-based CRT. Micke et al. [32] evaluated the influence of pre- and posttreatment CA 19-9 levels in 95 patients treated with CRT on outcome using the median CA 19-9 values of all patients as cutoff point. This group demonstrated that pretreatment levels of less than the median $(420 \mathrm{U} / \mathrm{ml})$ were significantly associated with a better objective tumor response ( 12.8 vs. $45.8 \%, \mathrm{p}=0.003)$ and overall survival (7.1 vs. 12.3 months, see table 1 ) in patients with unresectable disease. Patients with posttreatment CA 19-9 of less than the median $(293 \mathrm{U} / \mathrm{ml})$ had a longer median survival than patients with levels above the median. Additionally, patients with no decline in CA 19-9 during therapy had a significantly lower response rate. On multivariate analysis, pre- and posttreatment values as well as a tumor marker decrease during therapy were independent prognostic factors for overall survival (table 2) [32].

\section{CA 19-9 and Systemic Chemotherapy}

For patients with metastatic PC, palliative chemotherapy is the treatment of choice. Up till now, a systemic monotherapy with the nucleoside analog gemcitabine was regarded as the standard of care for patients with advanced stages of disease [33]. Combinations of gemcitabine with other cytostatic drugs in randomized trials have not yet shown any superior results concerning overall survival compared to single-agent gemcitabine [34, 35]. Adding the EGFR tyrosine kinase inhibitor erlotinib to gemcitabine seems to be the first combination regimen that significantly improves survival [36]. In patients treated with chemotherapy, the serum tumor marker CA 19-9 can potentially provide the following prognostic information: CA 19-9 values before and after treatment as well as CA 19-9 kinetics may have a prognostic impact, e.g. on survival, and dynamic changes of CA 19-9 levels during treatment may also be a biochemical surrogate marker for objective tumor response or progression (as determined by imaging criteria). In several clinical phase II trials, a reduction of CA 19-9 tumor marker levels under chemotherapy was described. A CA 19-9 decline $\geq 50 \%$ (according to baseline levels) was seen in $26-62 \%$ of all study patients treated with different chemotherapy regimens (as shown in table 3) [37-43].

\section{Pretreatment CA 19-9 Level}

As in surgery or CRT, in patients receiving systemic chemotherapy CA 19-9 baseline values may provide prognostic information. In a trial of Saad et al. [47] patients with pretreatment CA 19-9 levels below the median of the entire sample had a longer median survival than patients with levels above the median of $1,212 \mathrm{U} / \mathrm{ml}$ (table 1 ). On multivariate analysis, the pretreatment CA 19-9 level was an independent and highly significant predictor of survival $(\mathrm{p}=0.0005)$ and even a stronger one than a CA 199 response during chemotherapy $(\mathrm{p}=0.0497)$. Maisey et al. [52] recently confirmed these data on a large set of 154 patients receiving 5-FU- and/or gemcitabine-based chemotherapy for advanced PC. In their multivariate analysis, baseline CA 19-9 levels above or below the median value of $958 \mathrm{U} / \mathrm{ml}$ were found to be an independent prognostic factor for overall survival (HR 1.8, $\mathrm{p}=0.0004$, see table 1). Up to now, only one of all the published phase III trials reported - in an analysis for prognostic factors that in a group of 326 patients with advanced PC, baseline CA 19-9 levels ( $>350 \mathrm{U} / \mathrm{ml})$ were found to be a significant prognostic parameter on univariate as well as on multivariate analysis [53]. 
Table 3. CA 19-9 decline under chemotherapy in phase II trials

\begin{tabular}{|c|c|c|c|c|}
\hline Authors & $\begin{array}{l}\text { Chemotherapy } \\
\text { regimen }\end{array}$ & $\begin{array}{l}\text { CA } 19-9 \text { decrease } \\
\geq 50 \%, \% \text { of all patients }\end{array}$ & $\begin{array}{l}\text { DCR } \\
\%\end{array}$ & $\begin{array}{l}\text { TTP } \\
\text { months }\end{array}$ \\
\hline Philip et al. [37], 2001 & Gem/cisplatin & 62 & 62 & 5.4 \\
\hline Sherman and Fine [38], 2001 & Gem/docetaxel & 47 & 40 & n.a. \\
\hline Rocha Lima et al. [39], 2002 & Gem/irinotecan & 30 & 56 & 2.8 \\
\hline Stathopoulos et al. [40], 2004 & Gem/capecitabine & $39^{\mathrm{a}}$ & 60 & 6.5 \\
\hline Lee et al. [41], 2004 & Gem/uracil-tegafur & 57 & 41 & 4.2 \\
\hline Cantore et al. [42], 2004 & irinotecan/oxaliplatin & 26 & 33 & 4.1 \\
\hline Milella et al. [43], 2004 & celecoxib/5-FU & 33 & 24 & 2.0 \\
\hline
\end{tabular}

Gem = Gemcitabine; DCR = disease control rate, i.e. all patients achieving a remission or stable disease; TTP = median time to tumor progression; n.a. = not available.

${ }^{a}$ CA 19-9 decrease in patients with increased baseline levels (not defined as $\geq 50 \%$ ).

Table 4. Impact of CA19-9 response to chemotherapy on survival

\begin{tabular}{lcccc}
\hline Authors & $\mathrm{n}$ & $\begin{array}{c}\text { Definition of response } \\
\text { (CA 19-9 reduction), \% }\end{array}$ & \multicolumn{2}{c}{ Median overall survival, months } \\
\cline { 5 - 5 } & & & responder & nonresponder \\
\hline Ishii et al. [44], 1997 & 66 & $>50$ & 4.7 & 2.9 (p: n.a.) \\
Gogas et al. [45], 1998 & 35 & $\geq 15^{\mathrm{a}}$ & 11.1 & $6.2(\mathrm{p}=0.001)$ \\
Halm et al. [46], 2000 & 43 & $>20$ & 8.9 & $3.7(\mathrm{p}<0.001)$ \\
Saad et al. [47], 2002 & 28 & $\geq 50$ & 13.8 & $8.0(\mathrm{p}=0.0272)$ \\
Stemmler et al. [48], 2003 & 87 & $>50$ & 9.8 & $5.8(\mathrm{p}=0.022)$ \\
& & $\geq 15$ & 9.0 & $4.8(\mathrm{p}=0.017)$ \\
Ziske et al. [49], 2003 & 46 & $>20$ & 12.8 & $8.1(\mathrm{p}=0.006)$ \\
Sawaki et al. [50], 2004 & 40 & decreasing & 10.2 & $3.7(\mathrm{p}<0.001)$ \\
Ko et al. [51], 2005 & 76 & $>25$ & 9.61 & $4.64(\mathrm{p}<0.001)$ \\
& & $>50$ & 10.8 & $5.82(\mathrm{p}<0.001)$ \\
& & $>75$ & 12.0 & $6.0(\mathrm{p}<0.001)$ \\
\hline
\end{tabular}

n.a. = Not available.

a Definition of nonresponder: patients with a CA $19-9$ increase $>15 \%$ during therapy.

b Patients with decreasing CA 19-9 levels during treatment.
Impact of CA 19-9 Kinetics during Chemotherapy on Survival

Several clinical trials addressed the question of whether decreasing CA 19-9 serum levels during chemotherapeutic treatment have an influence on survival. Sawaki et al. [50] reported that patients treated with gemcitabine and decreasing CA 19-9 levels had a significantly longer survival than patients with increasing CA 19-9 levels during therapy (10.2 vs. 3.7 months, $\mathrm{p}<0.001)$. Previously, several investigators have tried to establish - similar to this observation - a definition of a biochemical treatment response (by reduction of CA 19-9 levels under chemotherapy), thereby correlating this response with median overall survival (as shown in table 4). Ishii et al. [44] in 1997 was the first author who defined a 'CA 19-9 responder' as a patient whose serum CA 19-9 level was reduced by more than $50 \%$ of the pretreatment level within 2 months after treatment. According to this definition, Stemmler et al. [48] showed that among patients with PC receiving a combination chemotherapy with gemcitabine and cisplatin CA 19-9 responders survived significantly longer than CA 19-9 nonresponders (295 vs. 174 days, $\mathrm{p}=0.022$ ). Saad et al. [47] defined a CA 19-9 responder using the same cutoff level (CA 19-9 decline $>50 \%$ ) but at any time after the initiation of treatment. In this study, patients with PC under treatment with single-agent gem- 
citabine that fulfilled the criteria of a CA 19-9 responder lived significantly longer than a CA 19-9 nonresponder (13.8 vs. 8 months, $\mathrm{p}=0.0272$ ). The study of Ko et al. [51] recently showed that a CA 19-9 decline of $>25,>50$ as well as $>75 \%$ during therapy is able to define subgroups of patients with a significant survival benefit compared to patients not responding with a CA 19-9 decrease under chemotherapy with fixed-dose rate gemcitabine.

In a retrospective study of 35 patients treated with the ECF regimen (epirubicin, cisplatin, 5-FU), a significant difference in median survival for patients with a CA 19-9 decrease $>15 \%$ compared to patients with a CA 19-9 increase $>15 \%$ on two consecutive occasions 3 weeks apart (333 vs. 185 days, $\mathrm{p}=0.001$ ) was observed [45]. Other groups used a CA $19-9$ decrease of $>20 \%$ of pretreatment levels after 8 weeks of treatment as a cutoff point to define a group of patients classified as CA 19-9 responders. Patients with advanced PC and a CA $19-9$ decrease $>20 \%$ of baseline CA 19-9 levels after 8 weeks of gemcitabine treatment had a significantly longer median survival than patients with a rise or a decrease $\leq 20 \%$ (268 vs. 110 days, $\mathrm{p}<0.001$ ) in a study published by Halm et al. [46] in 2000 . These results (with the same definition of a CA 19-9 response) were confirmed by Ziske et al. [49] and in both studies, not only median survival was significantly different between the two groups but also median time to progression. Additionally, both authors reported that on multivariate analysis, the response of CA $19-9>20 \%$ during gemcitabine chemotherapy was a strong and independent predictor of survival $[46,49]$. This observation was also confirmed by Maisey et al. [52] with their data showing that a fall of $20 \%$ in CA 19-9 levels following the start of a gemcitabine- and/or 5-FU-based chemotherapy is an independent prognostic factor for overall survival (HR 1.9, CI 1.1-3.4, $\mathrm{p}=0.019$ ) on multivariate analysis. Thus, not only pretreatment CA 19-9 levels but also a CA 19-9 response under chemotherapy may serve as an independent prognostic factor regarding survival.

\section{Correlation of CA 19-9 Changes during}

Chemotherapy with Objective Tumor Response

Up to now, it has not been clearly defined whether CA 19-9 changes may be used as a parameter to monitor treatment response in patients with PC treated with chemotherapy. Several studies reported that patients with an objective (radiographic) tumor response also often show a decline in serum CA 19-9 levels. But not all patients objectively responding to therapy do so and especially in patients with stable or progressive disease, the additional prognostic information obtained by serum tumor mark- er kinetics is unclear. In patients treated with gemcitabine and cisplatin, 8 out of 10 who achieved a radiological CR or PR had a decline of CA $19-9>50 \%$ during treatment, which was also seen in 10 out of 15 patients with disease stabilization and in 5 out of 12 patients (42\%) with progressive disease [37]. Heinemann et al. [54] reported 21 patients treated with the same combination regimen: in this study all 4 patients with CR reached normal CA 19-9 values during the course of treatment and all patients with PR showed a CA 19-9 decrease. Among patients with SD, CA 19-9 transiently decreased in 7/8 patients and in the group with PD CA 19-9 initially increased in $4 / 5$ patients. In the updated data from this trial, all patients with $C R$ and $10 / 11$ patients with $P R$ were considered CA 19-9 responders (defined by a decline $>50 \%$ within 2 months of treatment), but also 12 out of 27 patients with $\mathrm{PD}$ on imaging techniques showed a biochemical response characterized by decreases in CA 19-9 levels (8 of them qualified as CA 19-9 responders). This subgroup of patients (with PD on imaging and CA 19-9 response) had a significantly longer median survival than CA 19-9 nonresponders with PD (247 vs. 142 days, $\mathrm{p}=$ $0.04)$. The sensitivity of correlating CA $19-9$ response and CT findings in this study was $93.3 \%$ with only a low specificity of $53.2 \%$, respectively. The positive predictive value of a CA $19-9$ response was $32.5 \%$ with a high negative predictive value of $97.1 \%$ [48]. Comparable results were obtained from two trials with single-agent gemcitabine treatment in patients with PC. All patients with CR or PR were classified as CA 19-9 responder (defined by a decrease $>20 \%$ after 8 weeks of chemotherapy), but in both trials a CA 19-9 response in patients with PD on imaging modalities (2/7 and $2 / 20$, respectively) was also seen [ 46 , 49].

In two clinical phase II and III trials evaluating the efficacy of the irinotecan/gemcitabine combination in the treatment of advanced PC, CA 19-9 monitoring - as a potential parameter for treatment efficacy - was included. In the phase II part with 44 patients, Rocha Lima et al. [39] found a significant correlation between proportional changes in CA 19-9 and radiological changes of the tumor with regard to the extent of change $(r=0.67)$ and also a good correlation between CA 19-9 progression and time to progression $(r=0.89)$ with CA $19-9$ progression preceding radiographic progression in most of the patients. The overall diagnostic accuracy (determined in the phase III trial) of CA 19-9 values in the prediction of tumor response or progression was low (57.6 and 59.0\%, respectively). Nevertheless, a CA 19-9 decline of less than $50 \%$ may predict a lack of objective treatment response 
(negative predictive value: $94.8 \%$ ) and a CA $19-9$ progression (increase $>50 \%$ ) may be predictive for objective disease progression (positive predictive value: $82.8 \%$ ) [55]. Previously, Gogas et al. [45] reported a negative predictive value of serial CA 19-9 kinetics for an objective partial response, stable disease or progressive disease of 87,41 and $90 \%$, respectively.

\section{Discussion}

Up to now, CA 19-9 has been the most widely used 'standard' serum tumor marker in patients with PC. It has quite a good diagnostic sensitivity and specificity [for review, see Steinberg [9]) with some known limitations, e.g. in patients with (benign) jaundice, pancreatitis or other gastrointestinal malignancies. For monitoring patients under therapy and for assessing prognosis, the routine use of CA 19-9 is not generally recommended at present [56]. Nevertheless, there are several studies showing that CA 19-9 has the potential to become a clinically useful prognostic marker in patients with PC. In patients undergoing surgery for PC especially the decline of elevated baseline levels after operation may help to identify patients with a prolonged disease-free and overall survival [19]. Thus, CA 19-9 can provide useful additional information in therapeutic decision, e.g. in patients with a lack of CA 19-9 decline after tumor resection as candidates for different adjuvant treatment strategies.

In patients treated with CRT, pre- and posttreatment CA 19-9 levels show an association with overall survival $[28,31,32]$. Whether a biochemical treatment response, defined by declining CA 19-9 values under therapy, has an influence on survival is not yet clear. In the study of Micke et al. [32] a decrease of the serum tumor marker CA 19-9 during therapy was an independent prognostic factor regarding survival on multivariate analysis, but Wilkowski et al. [30] in their study could not confirm that the biochemical response status has an influence on survival.

Similar to the observations in patients treated with surgery or CRT, pretreatment CA 19-9 baseline values were found to have a strong and independent prognostic significance for patients treated with systemic chemotherapy $[47,52,53]$. But as shown in table 1 , different clinical studies reported a wide discrepancy for a CA 19-9 cutoff level (200-1,212 U/ml) that makes identifying prognostic relevant subgroups possible. Most investigators defined the cutoff point (that separates the two subgroups with a different survival prognosis in each individual trial) as the median of the CA 19-9 serum values in their patient population. Thus, it is not surprising that in clinical trials that included patients with resectable disease $[16,17]$ lower median CA 19-9 cutoff points were defined than in studies that enrolled only patients with an advanced stage of disease $[47,52]$. In order to achieve a unique definition of (individualized and stage-adapted) cutoff points that will help to separate different prognostic subgroups, a controlled prospective evaluation is necessary. Only a prospective clinical trial that defines fixed CA 19-9 cutoff points (e.g. 1,000 U/ml for patients with metastatic disease) will be able to lead to a unique definition of this clinical relevant issue.

Furthermore, several studies clearly showed that patients classified as CA 19-9 responders to chemotherapy have a significantly longer median survival than CA 19-9 nonresponders (table 4). Nevertheless, the role of CA 19-9 in monitoring (objective) treatment efficacy remains unclear. In most cases, patients with an objective complete or partial remission also show a biochemical treatment efficacy by decreasing CA 19-9 levels under chemotherapy. Especially the negative predictive value of CA 19-9 kinetics for a remission or for tumor progression seems to be good with levels up to $90 \%[48,55]$, but with certain limitations regarding the overall diagnostic accuracy of CA 19-9 values in the prediction of tumor response or progression [55]. Especially in patients with stable or progressive disease, the additional prognostic information of serial CA 19-9 changes is not defined. However, it was demonstrated that patients classified as CA 19-9 responders and with a documented disease progression on conventional imaging techniques had a significantly longer survival than CA 19-9 nonresponders with progressive disease [48]. It seems that a biochemical treatment effect has a prognostic meaning regarding survival, despite a documented disease progression on imaging. Thus, therapeutic consequences may also occur, e.g. that such a subgroup of patients may benefit from active second-line treatment regimens. Nevertheless, a CA 19-9 monitoring in patients with advanced PC undergoing palliative chemotherapy should still be regarded as one additional tool - besides imaging and clinical condition of the patient for treatment decisions.

\section{Future Perspectives}

As a first step for standardization, a unique assay should be used in clinical studies incorporating CA 19-9 measurements in order to generate comparable and reliable results. A standardized CA 19-9 measurement should 
also be included (e.g. as a secondary endpoint) in future phase III trials in order to confirm the data summarized here on the level of randomized trials with a large number of patients. In fact, only one phase III trial has reported CA 19-9 values as a prognostic factor up to now [53]. Regarding the obvious prognostic impact of CA 19-9 baseline levels in patients receiving systemic chemotherapy, a use of this tumor marker as a parameter of stratification in phase III trials appears to be justified.

Furthermore, a unique definition of a 'CA 19-9 response' is also required for monitoring treatment efficacy with CA 19-9 as a (biochemical) surrogate marker. A 'CA 19-9 responder' must not necessarily be defined as a patient with a CA $19-9$ reduction of at least $50 \%$ of the pretreatment level within 2 months after the initiation of treatment $[44,47]$. Several other groups clearly showed that also a CA 19-9 reduction with cutoff levels of 15, 20 or $25 \%$ was able to identify subgroups of patients with a prognostic favorable outcome regarding median overall survival (see table 4). Thus, a cutoff point of a CA 19-9 decline of more than $20 \%$ after 8 weeks of chemotherapeutic treatment seems to be sufficient to define a (clinically relevant) subgroup of patients with a prolonged survival. Based on the data summarized here, we recommend this definition of a 'CA 19-9 responder' for further prospective chemotherapeutic trials in patients with advanced PC. Despite these efforts regarding standardization, it still remains important to remember other limitations of serum tumor markers when monitoring a patient with PC: not only the alteration of CA 19-9 levels by different pathological processes and conditions (especially jaundice) but also the theoretical possibility of novel agents (e.g. monoclonal antibodies) interfering with the CA 19-9 assay must be considered carefully.
Tumor markers and other biological markers have the potential to become useful diagnostic and prognostic parameters in the treatment of patients with cancer. Serum tumor markers are easy to sample and their determination is - compared e.g. to radiological imaging modalities - cost effective and quick. In PC, new markers like genetic alterations and also other serum tumor antigens (e.g. CA 72-4 or CA 242) must be further investigated and both their diagnostic and prognostic role must be defined by comparative studies with CA $19-9$ [57, 58]. Pretreatment alterations (like S100A6 [59]) as well as kinetics of novel apoptotic markers like nucleosomes [60] during therapy may have prognostic significance, and thus may serve as helpful tools in therapeutic decisions in this harmful disease. Additionally, different laboratory methods for the determination of systemic inflammatory responses (e.g. by cytokines or C-reactive protein) in patients with $\mathrm{PC}$ will probably also serve as relevant predictors of outcome $[61,62]$.

\section{Conclusion}

The serum tumor marker CA 19-9 has the potential to permit both the assessment of prognosis and monitoring of patients with pancreatic adenocarcinoma. In patients with advanced disease, pretreatment CA 19-9 levels can provide prognostic information regarding overall survival and a decline of CA 19-9 ('CA 19-9 responder') under chemotherapy may serve as a useful additional tool for the go/no-go question in treatment decisions. A standardized measurement of CA 19-9 and a unique definition of CA 19-9 response to treatment are strongly recommended for further prospective trials.

\section{References}

1 Goldstein D, Carroll S, Apte M, et al: Modern management of pancreatic carcinoma. Intern Med J 2004;34:475-481.

2 van Custem E, van der Velde H, Karasek P, et al: Phase III trial of gemcitabine plus tipifarnib compared with gemcitabine plus placebo in advanced pancreatic cancer. J Clin Oncol 2004;22:1430-1438.

3 Ueno H, Okada S, Okusaka T, et al: Prognostic factors in patients with metastatic pancreatic adenocarcinoma receiving systemic chemotherapy. Oncology 2000;59: 296-301.
4 Brambs HJ, Claussen CD: Pancreatic and ampullary carcinoma. Ultrasound, computed tomography, magnetic resonance imaging and angiography. Endoscopy 1993;25: 58-68.

5 Anderson JS, Burris HA, Casper E, et al: Development of a new system for assessing clinical benefit for patients with advanced pancreatic cancer (abstract). Proc ASCO 1994; 13:1600.

6 Koprowski H, Steplewski Z, Mitchell K, et al: Colorectal carcinoma antigens detected by hybridoma antibodies. Somatic Cell Genet 1979;5:957-972.
7 Koprowski H, Herlyn M, Steplewski Z, et al: Specific antigen in serum of patients with colon carcinoma. Science 1981;212:53-55.

8 Tempero MA, Uchida E, Takasaki H, et al: Relationship of carbohydrate antigen 19-9 and Lewis antigens in pancreatic cancer. Cancer Res 1987;47:5501-5503.

9 Steinberg W: The clinical utility of the CA 19-9 tumor-associated antigen. Am J Gastroenterol 1990;85:350-355.

10 Safi F, Schlosser W, Kolb G, et al: Diagnostic value of CA 19-9 in patients with pancreatic cancer and nonspecific gastrointestinal symptoms. J Gastrointest Surg 1997;1:106112. 
11 Jiang XT, Tao HQ, Zou SC: Detection of serum tumor markers in the diagnosis and treatment of patients with pancreatic cancer. Hepatobiliary Pancreat Dis Int 2004;3:464468.

12 Bekaii-Saab TS, Cowgill SM, Burak WE, et al: Diagnostic accuracy of serum CA 19-9 in predicting malignancy in patients undergoing pancreatic resection (abstract). Proc ASCO 2004;22:4210.

13 Tanaka N, Okada S, Ueno H, et al: The usefulness of serial changes in serum CA 19-9 levels in the diagnosis of pancreatic cancer. Pancreas 2000;20:378-381.

14 Ni XG, Bai XF, Mao YL, et al: The clinical value of serum CEA, CA 19-9 and CA 242 in the diagnosis and prognosis of pancreatic cancer. Eur J Surg Oncol 2005;31:164-169.

15 Glenn J, Steinberg WM, Kurtzman SH, et al: Evaluation of the utility of a radioimmunoassay for serum CA 19-9 levels in patients before and after treatment of carcinoma of the pancreas. J Clin Oncol 1988;6:462-468.

16 Lundin J, Roberts PJ, Kuusela P, et al: The prognostic value of preoperative serum levels of CA 19-9 and CEA in patients with pancreatic cancer. Br J Cancer 1994;69:515-519.

17 Sperti C, Pasquali C, Catalini S, et al: CA 199 as a prognostic index after resection for pancreatic cancer. J Surg Oncol 1993;52:137141.

18 Sakar B, Aykan NF, Saglam S, et al: Longterm survival after curative resection for pancreatic ductal adenocarcinoma (abstract). Proc ASCO 2004;22:4227.

19 Montgomery RC, Hoffmann JP, Riley LB, et al: Prediction of recurrence and survival by post-resection CA 19-9 values in patients with adenocarcinoma of the pancreas. Ann Surg Oncol 1997;4:551-556.

20 Safi F, Schlosser W, Falkenrecht S, et al: Prognostic value of CA 19-9 serum course in pancreatic cancer. Hepatogastroenterology 1998;45:253-259.

21 Tian F, Appert HE, Myles J, et al: Prognostic value of serum CA 19-9 levels in pancreatic adenocarcinoma. Ann Surg 1992;215:350355.

22 Willet CG, Daly WJ, Warshaw AL: CA 19-9 is an index of response to neoadjunctive chemoradiation therapy in pancreatic cancer. Am J Surg 1996;172:350-352.

23 Wolff RA, Ayers GD, Crane CH, et al: Serum CA 19-9 levels in patients receiving preoperative gemcitabine (gem)-based chemoradiation for respectable pancreatic adenocarcinoma (abstract). Proc ASCO 2003;22: 1071.

24 Nishida K, Kaneko T, Yoneda M, et al: Doubling time of serum CA 19-9 in the clinical course of patients with pancreatic cancer and its significant association with prognosis. J Surg Oncol 1999;71:140-146.
25 Neoptolemos JP, Stocken DD, Friess H, et al: A randomized trial of chemoradiotheray and chemotherapy after resection of pancreatic cancer. N Engl J Med 2004;350:12001210.

26 Wilkowski R, Thoma M, Duhmke E, et al: Concurrent chemoradiotherapy with gemcitabine and cisplatin after incomplete (R1) resection of locally advanced pancreatic carcinoma. Int J Radiat Oncol Biol Phys 2004; 58:768-772.

27 Wilkowski R, Thoma M, Schauer R, et al: Effect of chemoradiotherapy with gemcitabine and cisplatin on locoregional control in patients with primary inoperable pancreatic cancer. World J Surg 2004;28:1011-1018.

28 Katz A, Hanlon A, Lanciano R, et al: Prognostic value of CA 19-9 levels in patients with carcinoma of the pancreas treated with radiotherapy. Int J Radiat Oncol Biol Phys 1998;41:393-396.

29 Okusaka T, Okada S, Sato T, et al: Tumor markers in evaluating the response to radiotherapy in unresectable pancreatic cancer. Hepatogastroenterology 1998;45:867-872.

30 Wilkowski R, Stieber P, Nolte A, et al: The prognostic importance of the CA 19-9 tumor marker and its use as a biochemical indicator of treatment response during (chemo-)radiotherapy of locally advanced pancreatic cancer (abstract). Proc ASCO 2004;22:4177.

31 Ikeda M, Okada S, Tokuuye K, et al: Prognostic factors in patients with locally advanced pancreatic carcinoma receiving chemoradiotherapy. Cancer 2001;91:490-495.

32 Micke O, Bruns F, Kurowski R, et al: Predictive value of carbohydrate antigen 19-9 in pancreatic cancer treated with radiochemotherapy. Int J Radiat Oncol Biol Phys 2003; 57:90-97.

33 Burris HA, Moore MJ, Andersen J, et al: Improvements in survival and clinical benefit with gemcitabine as first-line therapy for patients with advanced pancreas cancer: a randomized trial. J Clin Oncol 1997; 15:24032413.

34 Heinemann V: Gemcitabine in the treatment of advanced pancreatic cancer: a comparative analysis of randomized trials. Semin Oncol 2002;29(suppl 20):9-16.

35 Heinemann V: Present and future treatment of pancreatic cancer. Semin Oncol 2002. 29(suppl 9):23-31.

36 Moore MJ, Goldstein D, Hamm J, et al: Erlotinib plus gemcitabine compared to gemcitabine alone in patients with advanced pancreatic cancer. A phase III trial of the $\mathrm{Na}$ tional Cancer Institute of Canada Clinical Trials Group (NCIC-CTG) (abstract). Proc ASCO 2005;23:1.

37 Philip PA, Zalupski MM, Vaitkevicius VK, et al: Phase II study of gemcitabine and cisplatin in the treatment of patients with advanced pancreatic carcinoma. Cancer 2001; 92:569-577.
38 Sherman WH, Fine RL: Combination gemcitabine and docetaxel therapy in advanced adenocarcinoma of the pancreas. Oncology 2001;60:316-321.

39 Rocha Lima CMS, Savarese D, Bruckner H, et al: Iriontecan plus gemcitabine induces both radiographic and CA 19-9 tumor marker responses in patients with previously untreated advanced pancreatic cancer. J Clin Oncol 2002;20:1182-1191.

40 Stathopoulos GP, Syrigos K, Polyzos A, et al: Front-line treatment of inoperable or metastatic pancreatic cancer with gemcitabine and capecitabine: an intergroup, multicenter, phase II study. Ann Oncol 2004;15: 224-229.

41 Lee J, Park JO, Kim WS, et al: Phase II study of gemcitabine combined with uracil-tegafur in metastatic pancreatic cancer. Oncology 2004;66:32-37.

42 Cantore M, Rabbi C, Fiorentini G, et al: Combined irinotecan and oxaliplatin in patients with advanced pre-treated pancreatic cancer. Oncology 2004;67:93-97.

43 Milella M, Gelibter A, Di Cosimo S, et al: Pilot study of celecoxib and infusional 5-fluorouracil as second-line treatment for advanced pancreatic carcinoma. Cancer 2004; 101:133-138.

44 Ishii H, Okada S, Sato T, et al: CA 19-9 in evaluating the response to chemotherapy in advanced pancreatic cancer. Hepatogastroenterology 1997;44:279-283.

45 Gogas H, Lofts FJ, Evans TRJ, et al: Are serial measurements of CA 19-9 useful in predicting response to chemotherapy in patients with inoperable adenocarcinoma of the pancreas? Br J Cancer 1998;77:325-328.

46 Halm U, Schumann T, Schiefeke I, et al: Decrease of CA 19-9 during chemotherapy with gemcitabine predicts survival time in patients with advanced pancreatic cancer. $\mathrm{Br} \mathrm{J}$ Cancer 2000;82:1013-1016.

47 Saad ED, Machado MC, Wajsbrot D, et al: Pretreatment CA 19-9 level as a prognostic factor in patients with advanced pancreatic cancer treated with gemcitabine. Int J Gastrointest Cancer 2002;32:35-41.

48 Stemmler J, Stieber P, Szymala AM, et al: Are serial CA 19-9 kinetics helpful in predicting survival in patients with advanced or metastatic pancreatic cancer treated with gemcitabine and cisplatin? Onkologie 2003;26: 462-467.

49 Ziske C, Schlie C, Gorschlüter M, et al: Prognostic value of CA 19-9 levels in patients with inoperable adenocarcinoma of the pancreas treated with gemcitabine. Br J Cancer 2003; 89:1413-1417.

50 Sawaki A, Katsurahara M, Okubo K, et al: Can decreasing the serum level of CA 19-9 predict the survival benefit of gemcitabine for advanced pancreatic cancer? (abstract). Proc ASCO 2004;22:4208. 
51 Ko AH, Hwang J, Venook AP, et al: Serum CA 19-9 response as a surrogate marker for clinical outcome in patients receiving fixeddose rate gemcitabine for advanced pancreatic cancer. Br J Cancer 2005;93:195-199.

52 Maisey NR, Norman AR, Hill A, et al: CA 19-9 as a prognostic factor in inoperable pancreatic cancer: the implication for clinical trials. Br J Cancer 2005;93:740-743.

53 Louvet C, Labinaca R, Hammel P, et al: Gemcitabine in combination with oxaliplatin compared with gemcitabine alone in locally advanced or metastatic pancreatic cancer: results of a GERCOR and GISCAD phase III trial. J Clin Oncol 2005;23:35093516.
54 Heinemann V, Schermuly MM, Stieber P, et al: CA 19-9: a predictor of response in pancreatic cancer treated with gemcitabine and cisplatin. Anticancer Res 1999;19:2433-2436.

55 Rocha Lima CM, Green MR, Rotche R, et al: Irinotecan plus gemcitabine results in no survival advantage compared to gemcitabine monotherapy in patients with locally advanced or metastatic pancreatic cancer despite increased tumor response rate. J Clin Oncol 2004;22:3776-3783.

56 Tumour markers in gastrointestinal cancers - EGTM recommendations. European Group on Tumour Markers. Anticancer Res 1999;19:2811-2815.

57 Sawabu N, Watanabe H, Yamaguchi Y, et al: Serum tumor markers and molecular biological diagnosis in pancreatic cancer. Pancreas 2004;28:263-267.

58 Goggins M: Molecular markers of early pancreatic cancer. J Clin Oncol 2005;23:45244531 .
59 Vimalachandran D, Greenhalf W, Thompson C, et al: High nuclear S100A6 (calcyclin) is significantly associated with poor survival in pancreatic cancer patients. Cancer Res 2005;65:3218-3225.

60 Kremer A, Wilkowski R, Holdenrieder S, et al: Nucleosomes in pancreatic cancer patients during radiochemotherapy. Tumor Biol 2005;26:44-49.

61 Ebrahimi B, Tucker SL, Li D, et al: Cytokines in pancreatic carcinoma. Cancer 2004;101: 2727-2736.

62 Jamieson NB, Glen P, McMillan DC, et al: Systemic inflammatory response predicts outcome in patients undergoing resection for ductal adenocarcinoma head of pancreas. Br J Cancer 2005;92:21-23. 